\title{
On distance Laplacian spectrum of zero divisor graphs of the ring $\mathbb{Z}_{n}$
}

\author{
Pirzada S., Rather B.A., Chishti T.A.
}

\begin{abstract}
For a finite commutative ring $\mathbb{Z}_{n}$ with identity $1 \neq 0$, the zero divisor graph $\Gamma\left(\mathbb{Z}_{n}\right)$ is a simple connected graph having vertex set as the set of non-zero zero divisors, where two vertices $x$ and $y$ are adjacent if and only if $x y=0$. We find the distance Laplacian spectrum of the zero divisor graphs $\Gamma\left(\mathbb{Z}_{n}\right)$ for different values of $n$. Also, we obtain the distance Laplacian spectrum of $\Gamma\left(\mathbb{Z}_{n}\right)$ for $n=p^{z}, z \geq 2$, in terms of the Laplacian spectrum. As a consequence, we determine those $n$ for which zero divisor graph $\Gamma\left(\mathbb{Z}_{n}\right)$ is distance Laplacian integral.

Key words and phrases: Laplacian matrix, distance Laplacian matrix, commutative ring, zero divisor graph.
\end{abstract}

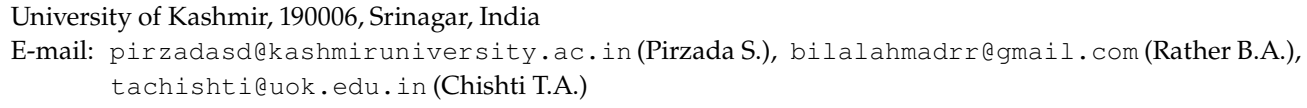

\section{Introduction}

In this paper, we consider only connected, simple and finite graphs. A graph is denoted by $G=G(V(G), E(G))$, where $V(G)=\left\{v_{1}, v_{2}, \ldots, v_{n}\right\}$ is its vertex set and $E(G)$ is its edge set. The order of $G$ is $n=|V(G)|$ and its size is $m=|E(G)|$. The set of vertices adjacent to $v \in V(G)$, denoted by $N(v)$, is the neighborhood of $v$. The degree of $v$, denoted by $d_{G}(v)$ (we simply write $d_{v}$ if it is clear from the context), means the cardinality of $N(v)$. A graph is regular if each of its vertices has the same degree. The adjacency matrix $A=\left(a_{i j}\right)$ of $G$ is a $(0,1)$-square matrix of order $n$ whose $(i, j)$-entry is equal to 1 if $v_{i}$ is adjacent to $v_{j}$ and equal to 0 , otherwise. Let $\operatorname{Deg}(G)=\operatorname{diag}\left(d_{1}, d_{2}, \ldots, d_{n}\right)$ be the diagonal matrix of vertex degrees $d_{i}=d_{G}\left(v_{i}\right), i=$ $1,2, \ldots, n$ associated to $G$. The matrices $L(G)=\operatorname{Deg}(G)-A(G)$ and $Q(G)=\operatorname{Deg}(G)+A(G)$ are respectively the Laplacian and the signless Laplacian matrices and these matrices are real symmetric and positive semi-definite. We take $0=\lambda_{n} \leq \lambda_{n-1} \leq \cdots \leq \lambda_{1}$ to be the Laplacian eigenvalues of $L(G)$.

In $G$, the distance between two vertices $u, v \in V(G)$, denoted by $d_{u v}$, is defined as the length of a shortest path between $u$ and $v$. The diameter of $G$ is the maximum distance between any two vertices of $G$. The distance matrix of $G$, denoted by $D(G)$, is defined as $D(G)=\left(d_{u v}\right)_{u, v \in V(G)}$. The transmission $\operatorname{Tr}_{G}(v)$ of a vertex $v$ is defined to be the sum of the distances from $v$ to all other vertices in $G$, i.e., $\operatorname{Tr}_{G}(v)=\sum_{u \in V(G)} d_{u v}$. A graph $G$ is said to be $k$-transmission regular if $\operatorname{Tr}_{G}(v)=k$, for each $v \in V(G)$. For any vertex $v_{i} \in V(G)$, the transmission $\operatorname{Tr}_{G}\left(v_{i}\right)$ is called the transmission degree, shortly denoted by $\operatorname{Tr}_{i}$ and the sequence $\left\{\operatorname{Tr}_{1}, \operatorname{Tr}_{2}, \ldots, \operatorname{Tr}_{n}\right\}$ is called the transmission degree sequence of the graph $G$. 
Let $\operatorname{Tr}(G)=\operatorname{diag}\left(\operatorname{Tr}_{1}, \operatorname{Tr}_{2}, \ldots, \operatorname{Tr}_{n}\right)$ be the diagonal matrix of vertex transmissions of $G$. Aouchiche M. and Hansen P. [2] introduced the Laplacian and the signless Laplacian for the distance matrix of a connected graph. The matrix $D^{L}(G)=\operatorname{Tr}(G)-D(G)$ is called the distance Laplacian matrix of $G$, while the matrix $D^{Q}(G)=\operatorname{Tr}(G)+D(G)$ is called the distance signless Laplacian matrix of $G$. These matrices are real symmetric and positive semi-definite, so we order the distance Laplacian eigenvalues as $\rho_{1} \geq \cdots \geq \rho_{n-1} \geq \rho_{n}=0$. More about $D^{L}(G)$ can be seen in $[2,3,7,11]$ and the references therein.

Let $R$ be a commutative ring with multiplicative identity $1 \neq 0$. A non-zero element $x \in R$ is called a zero divisor of $R$ if there exists a non-zero $y \in R$ such that $x y=0$. The zero divisor graph $\Gamma(R)$ of a commutative ring $R$ is the graph whose vertex set is the set of non-zero zero divisors of $R$ and two vertices $x$ and $y$ are adjacent if and only if $x y=0$ [1]. The zero divisor graph $\Gamma\left(\mathbb{Z}_{n}\right)$ is of order $n-\phi(n)-1$, where $\phi$ is Euler's totient function and $\mathbb{Z}_{n}$ is the integer modulo ring.

In a graph $G$, we write $x \sim y$ if vertices $x$ and $y$ are adjacent. We use standard notation, $K_{n}, K_{a, b}$, for complete graph and complete bipartite graph. Other undefined notations and terminology from algebraic graph theory, algebra and matrix theory can be found in $[4,6,8,10]$.

Investigation of spectral properties of matrices associated to graphs is always interesting and challenging. Since it is not always possible to obtain all the zeros of the characteristic polynomial of a matrix whose order is more than 4, therefore in most of the cases the study of spectra is restricted to certain families of graphs. Even at times the problem becomes difficult for a particular class of graphs. In this direction, we have considered the problem to investigate the distance Laplacian spectrum of zero divisor graphs associated to the ring $\mathbb{Z}_{n}$. We note that the graphs associated to different algebraic structures, for instance, power graphs and commuting graphs of groups, zero divisor graphs of rings have helped to solve several problems both in algebra and combinatorics. Adjacency eigenvalues of zero divisor graphs were first discussed by Young M. [9]. Laplacian, signless Laplacian and distance signless Laplacian spectral properties of zero divisor graphs were investigated in [5,11-13].

The rest of the paper is organized as follows. In Section 2, we discuss the distance Laplacian spectrum of the zero divisor graph $\Gamma\left(\mathbb{Z}_{n}\right)$ for some values of $n \in\left\{p q, p^{2} q,(p q)^{2}, p^{z}, z \geq 2\right\}$ and show that $\Gamma\left(\mathbb{Z}_{n}\right)$, for $n \in\left\{p^{z}, p q\right\}$, is distance Laplacian integral. We have used computational software Mathematica for computing approximate eigenvalues and characteristic polynomials of various matrices.

\section{The distance Laplacian spectrum of the zero divisor graphs}

The join of two graphs $G_{1}$ and $G_{2}$, denoted by $G_{1} \nabla G_{2}$, is the union of $G_{1}$ and $G_{2}$ together with edges from each vertex of $G_{1}$ to every vertex of $G_{2}$. The following result concerns with the distance Laplacian spectrum of the join of two graphs.

Theorem 1. Let $G_{1}$ and $G_{2}$ be graphs with $n_{1}$ and $n_{2}$ vertices, respectively. Let $\lambda_{1} \geq \lambda_{2} \geq \cdots \geq$ $\lambda_{n_{1}}=0$ and $\mu_{1} \geq \mu_{2} \geq \cdots \geq \mu_{n_{2}}=0$ be the Laplacian eigenvalues of $G_{1}$ and $G_{2}$. Then the distance Laplacian characteristic polynomial of $G_{1} \nabla G_{2}$ is

$$
P_{L}^{G_{1} \nabla G_{2}}(t)=t(t-n)\left(t-2 n+n_{2}+\lambda\right)^{n_{1}-1}\left(t-2 n+n_{1}+\mu\right)^{n_{2}-1},
$$

where $\lambda$ and $\mu$ are the non-zero Laplacian eigenvalues of $G_{1}$ and $G_{2}$, respectively. 
Proof. Let $G_{1} \nabla G_{2}$ be the join of the graphs $G_{1}$ and $G_{2}$. This is clearly a graph of diameter 2 . Let $V\left(G_{1}\right)=\left\{v_{1}, v_{2}, \ldots, v_{n_{1}}\right\}$ and $V\left(G_{2}\right)=\left\{u_{1}, u_{2}, \ldots, u_{n_{2}}\right\}$ be the vertex sets of $G_{1}$ and $G_{2}$, respectively. So $V(G)=V\left(G_{1}\right) \cup V\left(G_{2}\right)$. It is easy to see that $\operatorname{Tr}(v)=2 n-n_{2}-2-d(v)$, for each $v \in V\left(G_{1}\right)$ and $\operatorname{Tr}(u)=2 n-n_{2}-2-d(u)$, for each $u \in V\left(G_{2}\right)$. Label the vertices of $G_{1} \nabla G_{2}$, so that the first $n_{1}$ vertices are from $G_{1}$. With this labelling, the distance Laplacian matrix can be put in the form

$$
D^{L}\left(G_{1} \nabla G_{2}\right)=\left(\begin{array}{cc}
\left(2 n-n_{2}\right) I_{n_{1}}-2 J_{n_{1}}-L\left(G_{1}\right) & -J_{n_{1} \times n_{2}} \\
-J_{n_{2} \times n_{1}} & \left(2 n-n_{1}\right) I_{n_{2}}-2 J_{n_{2}}-L\left(G_{2}\right)
\end{array}\right),
$$

where $L\left(G_{2}\right), I_{n_{i}}$ and $J_{n_{1} \times n_{2}}$ are respectively the distance Laplacian matrix, identity matrix and the matrix whose each entry equals 1 for $i=1$, 2 . It is a well known fact that 0 the is Laplacian eigenvalue of $L\left(G_{i}\right)$ with the corresponding eigenvector $e_{n_{i}}=(\underbrace{1,1, \ldots, 1}_{n_{i}})^{T}$ for $i=1,2$, and all other eigenvectors of $L\left(G_{i}\right)$ are orthogonal to $e_{n_{i}}$. Let $x=\left(x_{i 1}, x_{i 2}, \ldots, x_{i n_{i}}\right)$ be the eigenvector of an arbitrary Laplacian eigenvalue $\lambda_{k}, 1 \leq k \leq n_{i}-1$, satisfying $e_{n_{i}}^{T} x=0$. Assigning $X=\left(\begin{array}{l}x \\ 0\end{array}\right)$ and noting that $J_{n_{1} \times n_{2}} x=0$, we obtain

$$
D^{L}\left(G_{1} \nabla G_{2}\right) X=\left(2 n-n_{2}-\lambda_{k}\right) X
$$

This implies that $2 n-n_{2}-\lambda_{k}, 1 \leq k \leq n_{1}-1$, is a distance Laplacian eigenvalue of $D^{L}\left(G_{1} \nabla G_{2}\right)$ with multiplicity $n_{1}-1$. Similarly, $2 n-n_{1}-\mu_{j}, 1 \leq j \leq n_{2}-1$, is a distance Laplacian eigenvalue of $D^{Q}\left(G_{1} \nabla G_{2}\right)$. The other two distance Laplacian eigenvalues $\{0, n\}$ are the zeros of the characteristic polynomial of the following quotient matrix

$$
\left(\begin{array}{cc}
n_{2} & -n_{2} \\
-n_{1} & n_{1}
\end{array}\right)
$$

This completes the proof.

If $K_{a, b}$ is the complete bipartite graph with partite sets of cardinality $a$ and $b$, then clearly $K_{a, b}=\bar{K}_{a} \nabla \bar{K}_{b}$. From Theorem 1, taking $n_{1}=a, n_{2}=b$, and $\lambda_{i}=\mu_{j}=0$ for each $i, j$, we observe that the distance Laplacian characteristic polynomial of $K_{a, b}$ is

$$
P_{L}^{K_{a, b}}(t)=t(t-n)(t-2 n+b)^{a-1}(t-2 n+a)^{b-1} .
$$

A complete split graph, denoted by $C S_{\omega, n-\omega}$, is the graph consisting of a clique on $\omega$ vertices and an independent set ${ }^{1}$ on the remaining $n-\omega$ vertices, such that each vertex of the clique is adjacent to every vertex of the independent set. Note that $C S_{\omega, n-\omega}=K_{\omega} \nabla \bar{K}_{n-\omega}$. Again, from Theorem 1, we observe that the distance Laplacian characteristic polynomial of $C S_{\omega, n-\omega}$ is

$$
P_{L}^{C S_{\omega, n-\omega}}(t)=t(t-n)^{\omega}(t-2 n+\omega)^{n-\omega-1} .
$$

This is because the Laplacian spectrum of $K_{\omega}$ is $\left\{\omega^{[\omega-1]}, 0\right\}$, apply Theorem 1 by taking $n_{1}=\omega, n_{2}=n-\omega, \lambda_{i}=\omega$ for $i=2, \ldots, \omega$, and $\mu_{j}=0$ for $j=2,3, \ldots, n-\omega$.

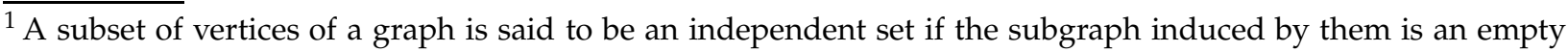
graph.
} 
The joined union of graphs is defined as follows [14]. Let $G(V, E)$ be a graph of order $n$ and $G_{i}\left(V_{i}, E_{i}\right)$ be graphs of order $m_{i}$, where $i=1, \ldots, n$. The joined union $G\left[G_{1}, \ldots, G_{n}\right]$ is the graph $H=(W, F)$ with

$$
W=\bigcup_{i=1}^{n} V_{i} \quad \text { and } \quad F=\bigcup_{i=1}^{n} E_{i} \cup \bigcup_{\left\{v_{i}, v_{j}\right\} \in E} V_{i} \times V_{j}
$$

In other words, the joined union is the union of graphs $G_{1}, \ldots, G_{n}$ together with the edges between the vertices $v_{i} \in V\left(G_{i}\right)$ and $v_{j} \in V\left(G_{j}\right)$ whenever $v_{i}$ and $v_{j}$ are adjacent in $G$.

The following result from [7] gives the distance Laplacian spectrum of $G\left[G_{1}, \ldots, G_{n}\right]$ in terms of the Laplacian spectrum of the graphs $G_{i}$, for $i=1,2, \ldots, n$.

Theorem 2 ([7]). Let $G$ be a graph of order $n$ having vertex set $V(G)=\left\{v_{1}, \ldots, v_{n}\right\}$. Let $G_{i}$ be a graph of order $m_{i}$ with Laplacian eigenvalues $\mu_{i 1} \geq \mu_{i 2} \geq \ldots \geq \mu_{i m_{i}}$, where $i=1,2, \ldots, n$. The distance Laplacian spectrum of the joined union $G\left[G_{1}, \ldots, G_{n}\right]$ consists of the eigenvalues $2 m_{i}-\mu_{i k}+\alpha_{i}$ for $i=1, \ldots, n$ and $k=1,2,3, \ldots, m_{i}-1$, where $\alpha_{i}=\sum_{k=1, k \neq i}^{n} m_{k} d_{G}\left(v_{i}, v_{k}\right)$. The remaining $n$ eigenvalues are given by the matrix

$$
M=\left(\begin{array}{cccc}
\alpha_{1} & -m_{2} d_{G}\left(v_{1}, v_{2}\right) & \ldots & -m_{n} d_{G}\left(v_{1}, v_{n}\right) \\
-m_{1} d_{G}\left(v_{2}, v_{1}\right) & \alpha_{2} & \ldots & -m_{n} d_{G}\left(v_{2}, v_{n}\right) \\
\vdots & \vdots & \ddots & \vdots \\
-m_{1} d_{G}\left(v_{n}, v_{1}\right) & -m_{2} d_{G}\left(v_{n}, v_{2}\right) & \ldots & \alpha_{n}
\end{array}\right)
$$

An integer $d$ is a proper divisor of $n$ if $d$ divides $n$, written as $d \mid n$, for $1<d<n$. Let $\mathrm{Y}_{n}$ be the simple graph with vertex set $\left\{d_{1}, d_{2}, \ldots, d_{t}\right\}$ and two distinct vertices in $Y_{n}$ are adjacent if and only if $n \mid d_{i} d_{j}$. If the prime power factorization of $n=p_{1}^{n_{1}} p_{2}^{n_{2}} \ldots p_{r}^{n_{r}}$, where $r, n_{1}, n_{2}, \ldots, n_{r}$ are positive integers and $p_{1}, p_{2}, \ldots, p_{r}$ are distinct prime numbers, the size of $Y_{n}$ is given by $\left|V\left(\mathrm{Y}_{n}\right)\right|=\prod_{i=1}^{r}\left(n_{i}+1\right)-2$. For $1 \leq i \leq t$, we consider the sets $A_{d_{i}}=\left\{x \in \mathbb{Z}_{n}:(x, n)=d_{i}\right\}$. We see that $A_{d_{i}} \cap A_{d_{j}}=\phi$, when $i \neq j$, implying that the sets $A_{d_{1}}, A_{d_{2}}, \ldots, A_{d_{t}}$ are pairwise disjoint and partitions the vertex set of $\Gamma\left(\mathbb{Z}_{n}\right)$ as $V\left(\Gamma\left(\mathbb{Z}_{n}\right)\right)=A_{d_{1}} \cup A_{d_{2}} \cup \cdots \cup A_{d_{t}}$. From definition of $A_{d_{i}}$, a vertex of $A_{d_{i}}$ is adjacent to a vertex of $A_{d_{j}}$ in $\Gamma\left(\mathbb{Z}_{n}\right)$ if and only if $n$ divides $d_{i} d_{j}$ for $i, j \in\{1,2, \ldots, t\}$.

The following result from [9] gives the cardinality of $A_{d_{i}}$.

Lemma 1 ([9]). Let $d_{i}$ be the proper divisor of $n$. Then

$$
\left|A_{d_{i}}\right|=\phi\left(\frac{n}{d_{i}}\right)
$$

for $1 \leq i \leq t$.

The next lemma from [5] shows that the induced subgraphs $\Gamma\left(A_{d_{i}}\right)$ of $\Gamma\left(\mathbb{Z}_{n}\right)$ are either cliques or their complements. 
Lemma 2 ([5]). The following hold.

(i) For $i \in\{1,2, \ldots, t\}$, the induced subgraph $\Gamma\left(A_{d_{i}}\right)$ of $\Gamma\left(\mathbb{Z}_{n}\right)$ on the vertex set $A_{d_{i}}$ is either a complete graph $K_{\phi\left(\frac{n}{d_{i}}\right)}$ or its complement $\bar{K}_{\phi\left(\frac{n}{d_{i}}\right)}$. Indeed, $\Gamma\left(A_{d_{i}}\right)$ is $K_{\phi\left(\frac{n}{d_{i}}\right)}$ if and only if $n$ divides $d_{i}^{2}$.

(ii) For $i, j \in\{1,2, \ldots, t\}$ with $i \neq j$, a vertex of $A_{d_{i}}$ is adjacent to either all or none of the vertices of $A_{d_{j}}$ in $\Gamma\left(\mathbb{Z}_{n}\right)$.

The following lemma shows that $\Gamma\left(\mathbb{Z}_{n}\right)$ is the joined union of certain complete graphs and null graphs.

Lemma 3 ([5]). Let $\Gamma\left(A_{d_{i}}\right)$ be the induced subgraph of $\Gamma\left(\mathbb{Z}_{n}\right)$ on the vertex set $A_{d_{i}}$ for $1 \leq i \leq t$. Then

$$
\Gamma\left(\mathbb{Z}_{n}\right)=\mathrm{Y}_{n}\left[\Gamma\left(A_{d_{1}}\right), \Gamma\left(A_{d_{2}}\right), \ldots, \Gamma\left(A_{d_{t}}\right)\right] .
$$

We consider an example to find the distance Laplacian spectrum with the help of Theorem 2. We know that $\Gamma\left(\mathbb{Z}_{n}\right)$ is a complete graph if and only if $n=p^{2}$ for some prime $p$. Further, the adjacency spectrum of $K_{\omega}$ and $\bar{K}_{\omega}$ on $\omega$ vertices are $\left\{\omega-1,-1^{[\omega-1]}\right\}$ and $\left\{0^{[\omega]}\right\}$, respectively. So, by Theorem 2 , out of $n-\phi(n)-1$ number of distance Laplacian eigenvalues of $\Gamma\left(\mathbb{Z}_{n}\right), n-\phi(n)-1-t$ of them are known to be positive integers. The remaining $t$ distance Laplacian eigenvalues of $\Gamma\left(\mathbb{Z}_{n}\right)$ will count from the zeros of the characteristic polynomial of the equitable quotient matrix $M$.

Example 1. Distance Laplacian eigenvalues of $\Gamma\left(\mathbb{Z}_{30}\right)$.

Let $n=30$. Then 2,3,5,6,10 and 15 are the proper divisors of $n$ and $\mathrm{Y}_{n}$ is the graph $G_{6}: 3 \sim 10 \sim 6 \sim 5,10 \sim 15 \sim 2$ and $6 \sim 15$, that is, $Y_{n}$ is the triangle graph having a pendent edge at each vertex of the triangle. Indexing the vertices by increasing proper divisor sequence and applying Lemma 3, we have

$$
\Gamma\left(\mathbb{Z}_{30}\right)=\mathrm{Y}_{30}\left[\bar{K}_{8}, \bar{K}_{4}, \bar{K}_{2}, \bar{K}_{4}, \bar{K}_{2}, K_{1}\right] .
$$

By Theorem 2, the distance Laplacian spectrum of $\Gamma\left(\mathbb{Z}_{30}\right)$ consists of the eigenvalues

$$
\left\{48^{[4]}, 47^{[7]}, 37^{[3]}, 33\right\}
$$

and the remaining six eigenvalues are the zeros of the characteristic polynomial of the following matrix

$$
\left(\begin{array}{cccccc}
31 & -12 & -6 & -8 & -4 & -1 \\
-24 & 42 & -6 & -8 & -2 & -2 \\
-24 & -12 & 46 & -4 & -4 & -2 \\
-16 & -8 & -2 & 29 & -2 & -1 \\
-16 & -4 & -4 & -4 & 29 & -1 \\
-8 & -8 & -4 & -4 & -2 & 26
\end{array}\right)
$$

The characteristic polynomial of matrix (1) is

$$
x^{6}-203 x^{5}+16167 x^{4}-631097 x^{3}+12078472 x^{2}-90732012 x
$$


and its approximated zeros are

$\{54.8984,52.8576,37.2458,31.1478,27.0505,0.0000\}$.

Now, we find the distance Laplacian spectrum of $\Gamma\left(\mathbb{Z}_{n}\right)$ for $n \in\left\{p q, p^{2} q,(p q)^{2}, p^{z}, z \geq 2\right\}$ with the help of Theorems 1 and 2. Consider $n=p q$, where $p$ and $q, p<q$, are distinct primes. We use either Theorem 1 or Theorem 2. By Lemmas 2 and 3, we have

$$
\Gamma\left(\mathbb{Z}_{p q}\right)=\mathrm{Y}_{p q}\left[\Gamma\left(A_{p}\right), \Gamma\left(A_{q}\right)\right]=K_{2}\left[\bar{K}_{\phi(p)}, \bar{K}_{\phi(q)}\right]=\bar{K}_{\phi(p)} \nabla \bar{K}_{\phi(q)}=K_{\phi(p), \phi(q)} .
$$

Lemma 4. The distance Laplacian spectrum of $\Gamma\left(\mathbb{Z}_{n}\right)$ consists of the eigenvalues $2 n-q+1$ with multiplicity $p-2$, the eigenvalue $2 n-p+1$ with multiplicity $q-2$, and the eigenvalues $\{0, n\}$.

Proof. Let $n=p q$, where $p$ and $q, p<q$, are distinct primes. Then the proper divisors of $n$ are $p$ and $q$, so $\mathrm{Y}_{p q}$ is $K_{2}$. By equation (2) and Theorem 1 the result follows.

The next result gives the distance Laplacian spectrum of $\Gamma\left(\mathbb{Z}_{p^{2} q}\right)$ for $n=p^{2} q$.

Lemma 5. The distance Laplacian spectrum of $\Gamma\left(\mathbb{Z}_{p^{2} q}\right)$ is

$$
\begin{aligned}
\left\{\left(3 p q+2 p^{2}-2 p-2 q\right)^{\left[p^{2}-p-1\right]},\right. & \left(p q+2 p^{2}-2 p\right)^{[p-2]},\left(2 p q+p^{2}-2 p-1\right)^{[q-2]} \\
& \left.\left(3 p^{2}+2 p q-4 p-1\right)^{p q-p-q}, x_{1} \geq x_{2} \geq x_{3} \geq x_{4}=0\right\}
\end{aligned}
$$

where $x_{1}, x_{2}, x_{3}$ are the non-zero zeros of the characteristic polynomial of the quotient matrix $M\left(P_{4}\right)$.

Proof. Let $n=p^{2} q$, where $p$ and $q$ are distinct primes. Since proper divisors of $n$ are $p, q, p q, p^{2}$, so $\mathrm{Y}_{p^{2} q}$ is the path $P_{4}: q \sim p^{2} \sim p q \sim p$. By Lemma 3, we have

$$
\Gamma\left(\mathbb{Z}_{p^{2} q}\right)=Y_{p^{2} q}\left[\Gamma\left(A_{q}\right), \Gamma\left(A_{P^{2}}\right), \Gamma\left(A_{p q}\right), \Gamma\left(A_{p}\right)\right]=P_{4}\left[\bar{K}_{\phi\left(p^{2}\right)}, \bar{K}_{\phi(q)}, K_{\phi(p)}, \bar{K}_{\phi(p q)}\right],
$$

with respective orders $m_{1}=\phi\left(p^{2}\right), m_{2}=\phi(q), m_{3}=\phi(p)$ and $m_{4}=\phi(p q)$. Further, we see that $\left(\alpha_{1}, \alpha_{2}, \alpha_{3}, \alpha_{4}\right)=\left(3 p q-p-2 q, 2 p q+p^{2}-2 p-2 q+1,2 p^{2}+p q-3 p, 3 p^{2}+2 q-2 p-3\right)$ and by Theorem 2, distance Laplacian spectrum of $\Gamma\left(\mathbb{Z}_{p^{2} q}\right)$ consists of the eigenvalues $3 p q+2 p^{2}-2 p-2 q, p q+2 p^{2}-2 p, 2 p q+p^{2}-2 p-1,3 p^{2}+2 p q-4 p-1$ with multiplicities $p^{2}-p-1, p-2, q-2, p q-p-q$, respectively. The remaining eigenvalues are given by the following of the equitable quotient matrix

$$
M\left(P_{4}\right)=\left(\begin{array}{cccc}
\alpha_{1} & -\phi(q) & -2 \phi(p) & -3 \phi(p q) \\
-\phi\left(p^{2}\right) & \alpha_{2} & -\phi(p) & -2 \phi(p q) \\
-2 \phi\left(p^{2}\right) & -\phi(q) & \alpha_{3} & -\phi(p q) \\
-3 \phi\left(p^{2}\right) & -2 \phi(q) & -\phi(p) & \alpha_{4}
\end{array}\right) .
$$

Clearly, each row sum of $M\left(P_{4}\right)$ is zero, so that 0 is its eigenvalue and the remaining eigenvalues are the non-zero zeros of the characteristic polynomial of $M\left(P_{4}\right)$. 
Lemma 6. The distance Laplacian spectrum of $\Gamma\left(\mathbb{Z}_{(p q)^{2}}\right)$ is

$$
\begin{aligned}
& \left\{\left(3 p^{2} q+2 p q^{2}+2 q-5 p-2 p q\right)^{\left[\phi\left(p q^{2}\right)-1\right]},\left(3 p^{2} q+2 p q^{2}-q-2 p^{2}-2 p q\right)^{\left[\phi\left(q^{2}\right)-1\right]}\right. \\
& \left(3 p^{2} q+2 p q^{2}-q-3 p q-1\right)^{\left[\phi\left(p^{2} q\right)-1\right]},\left(2 p^{2} q+3 p q^{2}+q-2 q^{2}-3 p q-1\right)^{\left[\phi\left(p^{2}\right)-1\right]} \\
& \left(2 p^{2} q+3 p q^{2}+p-6 q-4 p q\right)^{[\phi(p q)-1]},\left(2 p^{2} q+3 p q^{2}-p-q-p q\right)^{[\phi(p)-1]} \\
& \left.\left(p^{2} q+2 p q^{2}-2 p q-1\right)^{[\phi(q)-1]}\right\}
\end{aligned}
$$

and the zeros of the characteristic polynomial of the matrix $M\left(G_{7}\right)$ in (2).

Proof. Let $n=(p q)^{2}$, where $p$ and $q, p<q$, are distinct primes. Since the proper divisors of $n$ are $p, p^{2}, q, q^{2}, p q, p q^{2}$ and $p^{2} q$, so $\mathrm{Y}_{(p q)^{2}}$ is the graph $G_{7}: q \sim p^{2} q \sim q \sim p^{2} \sim p q^{2} \sim p$, $p^{2} q \sim p q$. We name the vertices in $G_{7}$ according to the proper divisor sequence, so by Lemma 3 , we have

$$
\begin{aligned}
\Gamma\left(\mathbb{Z}_{(p q)^{2}}\right) & =\mathrm{Y}_{(p q)^{2}}\left[\Gamma\left(A_{p}\right), \Gamma\left(A_{p^{2}}\right), \Gamma\left(A_{q}\right), \Gamma\left(A_{q^{2}}\right), \Gamma\left(A_{p q}\right), \Gamma\left(A_{p q^{2}}\right), \Gamma\left(A_{p^{2} q}\right)\right] \\
& =G_{7}\left[\bar{K}_{\phi\left(p q^{2}\right)}, \bar{K}_{\phi\left(q^{2}\right)}, \bar{K}_{\phi\left(p^{2} q\right)}, \bar{K}_{\phi\left(p^{2}\right)}, K_{\phi(p q)}, K_{\phi(p)}, K_{\phi(q)}\right] .
\end{aligned}
$$

By Theorem 2, we have

$$
\begin{aligned}
\left(\alpha_{1}, \alpha_{2}, \alpha_{3}, \alpha_{4}, \alpha_{5}, \alpha_{6}, \alpha_{7}\right)= & \left(3 p^{2} q+2 q^{2}-5 p, 3 p^{2} q+2 p q^{2}+q-2 p^{2}-2 p q,\right. \\
& 3 p q^{2}+2 p^{2}-2 p-q-p q-1, \\
& 2 p^{2} q+3 p q^{2}+2 p+q-2 p^{2}-2 q^{2}-3 p q-1, \\
& 2 p^{2} q+3 p q^{2}+2 p-5 q-5 p q-1,2 p^{2} q+p q^{2}-2 p-q-p q+1, \\
& \left.2 p^{2} q+p q^{2}-2 p-q\right) .
\end{aligned}
$$

Again, by Theorem 2, the distance Laplacian spectrum of $\Gamma\left(\mathbb{Z}_{(p q)^{2}}\right)$ consists of the eigenvalue $2 \phi\left(p q^{2}\right)+\alpha_{1}=3 p^{2} q+2 p q^{2}+2 q-5 p-2 p q$ with multiplicity $\phi\left(p q^{2}\right)-1$, the eigenvalue $3 p^{2} q+2 p q^{2}-q-2 p^{2}-2 p q$ with multiplicity $\phi\left(q^{2}\right)-1$, the eigenvalue $3 p^{2} q+2 p q^{2}-q-3 p q-1$ with multiplicity $\phi\left(p^{2} q\right)-1$, the eigenvalue $2 p^{2} q+3 p q^{2}+q-2 q^{2}-3 p q-1$ with multiplicity $\phi\left(p^{2}\right)-1$, the eigenvalue $2 p^{2} q+3 p q^{2}+p-6 q-4 p q$ with multiplicity $\phi(p q)-1$, the eigenvalue $2 p^{2} q+3 p q^{2}-p-q-p q$ with multiplicity $\phi(p)-1$, the eigenvalue $p^{2} q+2 p q^{2}-2 p q-1$ with multiplicity $\phi(q)-1$ and the remaining seven eigenvalues are given by the following matrix

$$
M\left(G_{7}\right)=\left(\begin{array}{ccccccc}
\alpha_{1} & -2 \phi\left(q^{2}\right) & -3 \phi\left(p^{2} q\right) & -3 \phi\left(p^{2}\right) & -3 \phi(p q) & -\phi(p) & -2 \phi(q) \\
-2 \phi\left(p q^{2}\right) & \alpha_{2} & -3 \phi\left(p^{2} q\right) & -\phi\left(p^{2}\right) & -3 \phi(p q) & -\phi(p) & -2 \phi(q) \\
-3 \phi\left(p q^{2}\right) & -3 \phi\left(q^{2}\right) & \alpha_{3} & -2 \phi\left(p^{2}\right) & -2 \phi(p q) & -2 \phi(p) & -\phi(q) \\
-3 \phi\left(p q^{2}\right) & -\phi\left(q^{2}\right) & -2 \phi\left(p^{2} q\right) & \alpha_{4} & -2 \phi(p q) & -2 \phi(p) & -\phi(q) \\
-3 \phi\left(p q^{2}\right) & -3 \phi\left(q^{2}\right) & -2 \phi\left(p^{2} q\right) & -2 \phi\left(p^{2}\right) & \alpha_{5} & -2 \phi(p) & -\phi(q) \\
-\phi\left(p q^{2}\right) & -\phi\left(q^{2}\right) & -2 \phi\left(p^{2} q\right) & -2 \phi\left(p^{2}\right) & -2 \phi(p q) & \alpha_{6} & -\phi(q) \\
-2 \phi\left(p q^{2}\right) & -2 \phi\left(q^{2}\right) & -\phi\left(p^{2} q\right) & -\phi\left(p^{2}\right) & -\phi(p q) & -\phi(p) & \alpha_{7}
\end{array}\right) .
$$

By using values of $\alpha_{i}^{\prime}$ s, we see that each row sum of the matrix $M\left(G_{7}\right)$ is zero, so 0 is its distance Laplacian eigenvalue and other six eigenvalues are the non-zero zeros of the characteristics polynomial of the above given matrix. 
Now, we find the distance Laplacian spectrum of $\Gamma\left(\mathbb{Z}_{n}\right)$ for $n=p^{z}, z \geq 3$. We consider $n=p^{2 m}$ and similar type of analysis can be done for $n=p^{2 m+1}$. If $n=p^{2 m}$, then the divisor sequence is $\left\{p, p^{2}, \ldots, p^{2 m-1}\right\}$ and vertex $p^{i}$ is adjacent to vertex $p^{j}$ in $Y_{p^{2 m}}$ for each $j \geq 2 m-i$ with $1 \leq i \leq 2 m-1$ and $i \neq j$. By Lemma 1 , order of $G_{i}$ is $m_{i}=\phi\left(p^{2 m-i}\right)$ for $i=1,2, \ldots, 2 m-1$. Also, for $i=1,2, \ldots, m-1, G_{i}=\bar{K}_{\phi\left(p^{2 m-i}\right)}$ and for $i=m, \ldots, 2 m-1, G_{i}=K_{\phi\left(p^{2 m-i}\right)}$. From Theorem 2 , the distance Laplacian spectrum of $\Gamma\left(\mathbb{Z}_{n}\right)$ consists of $\rho_{i}=2 p^{2 m-1}-p^{i}-1$, with respective multiplicities $\phi\left(p^{2 m-i}\right)-1$ for $i=1,2, \ldots, 2 m-1$, and the remaining eigenvalues are given by equitable quotient of $\Gamma\left(\mathbb{Z}_{n}\right)$. We observe that $\rho_{i}$ adds the missing entry in the definition of $\alpha_{i}$ in Theorem 2 .

Now, the remaining eigenvalues of the quotient matrix can be found in several ways. One way is to use the mathematical induction and show that the eigenvalues of quotient matrix are those of $\rho_{i}$ together with 0 and each with multiplicity 1 except when $i \neq m$. Another way is to construct $\Gamma\left(\mathbb{Z}_{n}\right)$ by using iteration join graphs as in [5] and use Theorem 1 continuously till we get all the eigenvalues. The natural way is to see that $\Gamma\left(\mathbb{Z}_{n}\right)$ is a graph of diameter two, so that distance Laplacian spectrum of $\Gamma\left(\mathbb{Z}_{n}\right)$ can be found in terms of spectrum of the Laplacian matrix. For that we need the following results.

Theorem 3 ([2]). Let $G$ be a connected graph on $n$ vertices with diameter at most two. Then the distance Laplacian spectrum of $G$ is

$$
2 n-\lambda_{n-1} \geq 2 n-\lambda_{n-2} \cdots \geq 2 n-\lambda_{1}>\rho_{n}=0 .
$$

A matrix of order $n$ with real entries is said to be integral, if all its eigenvalues are integers. Likewise, a graph $G$ is said to be integral if its associated matrix is integral.

A consequence of the above result is the following, whose proof is trivial.

Corollary 1. Let $G$ be graph on $n$ vertices with diameter at most two. Then $G$ is Laplacian integral if and only if $G$ is distance Laplacian integral.

Theorem 4 ([5]). Let $n=p^{z}$ where $p$ is prime and $z \geq 2$ is a positive integer. Then the following hold.

(i) If $z=2$, then the Laplacian spectrum of $\Gamma\left(\mathbb{Z}_{n}\right)$ is $\left\{(p-1)^{[p-2]}, 0\right\}$.

(ii) If $z=p^{2 m}$ for some positive integer $m \geq 2$, then the Laplacian spectrum of $\Gamma\left(\mathbb{Z}_{n}\right)$ consists of the eigenvalue 0 , the eigenvalue $p^{i}-1$ with multiplicity $\phi\left(p^{2 m-i}\right)$, where $i=2 m-1,2 m-2, \ldots, m+2, m+1, m-1, m-2, \ldots, 1$ and the eigenvalue $p^{m}-1$ with multiplicity $\phi\left(p^{m}\right)-1$.

(iii) If $z=p^{2 m+1}$ for some positive integer $m \geq 1$, then the distance Laplacian spectrum of $\Gamma\left(\mathbb{Z}_{n}\right)$ consists of the eigenvalue 0 , the eigenvalue $p^{i}-1$ with multiplicity $\phi\left(p^{2 m+1-i}\right)$, where $i=2 m, 2 m-1, \ldots, m+2, m+1, m-1, m-2, \ldots, 1$ and the eigenvalue $p^{m}-1$ with multiplicity $\phi\left(p^{m+1}\right)-1$. 
Theorem 5. Let $n=p^{z}$ where $p$ is prime and $z \geq$ is a positive integer. Then the following hold.

(i) If $z=2$, then the distance Laplacian spectrum of $\Gamma\left(\mathbb{Z}_{n}\right)$ is $\left\{(p-1)^{[p-2]}, 0\right\}$.

(ii) If $z=p^{2 m}$ for some positive integer $m \geq 2$, then the distance Laplacian spectrum of $\Gamma\left(\mathbb{Z}_{n}\right)$ consists of the simple eigenvalue 0 , the eigenvalue $2 p^{2 m-1}-p^{2 m-i}-1$ with multiplicity $\phi\left(p^{i}\right)$ for $i=2 m-1,2 m-2, \ldots, m+1, m-1, \ldots, 2,1$ and the eigenvalue $2 p^{2 m-1}-p^{m}-1$ with multiplicity $\phi\left(p^{m}\right)-1$.

(iii) If $z=p^{2 m}$ for some positive integer $m \geq 2$, then the distance Laplacian spectrum of $\Gamma\left(\mathbb{Z}_{n}\right)$ consists of the simple eigenvalue 0 , the eigenvalue $2 p^{2 m-1}-p^{2 m-i}-1$ with multiplicity $\phi\left(p^{i}\right)$ for $i=2 m, 2 m-1, \ldots, m+1, m-1, \ldots, 2,1$ and the eigenvalue $2 p^{2 m-1}-p^{m}-1$ with multiplicity $\phi\left(p^{m+1}\right)-1$.

Proof. (i) Since $\Gamma\left(\mathbb{Z}_{p^{2}}\right)=\Gamma\left(A_{p}\right)$ is the complete graph $K_{p-1}$ for any prime $p$, so the result follows for $p>2$.

The other cases follow from Theorem 3 and Theorem 4 and using the fact that order of $\mathbb{Z}_{n}$ is $n=\phi(n)-1$.

Now, for $n=p^{3}$, by Lemma 3 we have

$$
\Gamma\left(\mathbb{Z}_{p^{3}}\right)=\mathrm{Y}_{p^{3}}\left[\Gamma\left(A_{p}\right), \Gamma\left(A_{p^{2}}\right)\right]=K_{2}\left[\bar{K}_{\phi\left(p^{2}\right)}, \bar{K}_{\phi(p)}\right]=\bar{K}_{p(p-1)} \nabla K_{p-1} .
$$

This implies that $\Gamma\left(\mathbb{Z}_{p^{3}}\right)$ is a complete split graph of order $p^{2}-1$, having independent set of cardinality $p(p-1)$ and clique of size $p-1$. By Theorem 1, distance Laplacian spectrum of $\Gamma\left(\mathbb{Z}_{n}\right)$ is $\left\{\left(2 p^{2}-p+1\right)^{\left[p^{2}-p-1\right]},\left(p^{2}-1\right)^{[p-2]}, 0\right\}$.

From the facts and discussions above, we have the following observation.

Corollary 2. $\Gamma\left(\mathbb{Z}_{n}\right)$ is distance Laplacian integral if and only if $n$ is prime power or product of two distinct primes.

Proof. If $n$ is either prime power or product of two distinct primes, then by Lemma 4 and Theorem 5 , it follows that the distance Laplacian eigenvalues of $\Gamma\left(\mathbb{Z}_{n}\right)$ are integers. Also, by Lemma 5 , for $p=2^{2}, \Gamma\left(\mathbb{Z}_{4 q}\right)$ is the complete bipartite graph and its distance Laplacian eigenvalues are integers. For other way round, if $n$ is the product of three primes, then by Example 1, we get some non-integer distance Laplacian eigenvalues of $\Gamma\left(\mathbb{Z}_{p q r}\right)$, where $p, q, r, p<q<r$ are primes. More generally, if $n=p_{1}^{n_{1}} p_{2}^{n_{2}} \ldots p_{r}^{n_{r}}$, where $r, n_{1}, \ldots, n_{r}$ are non-negative integers and $p_{i}, i=1,2, \ldots, r$, are primes, then for $r \geq 3, \Gamma\left(\mathbb{Z}_{n}\right)$ contains the triangle $\frac{n}{p_{3}^{n_{3}}} \sim \frac{n}{p_{2}^{n_{2}}} \sim \frac{n}{p_{1}^{n_{1}}} \sim \frac{n}{p_{3}^{n_{3}}}$, which implies that $\Gamma\left(\mathbb{Z}_{n}\right)$ is not complete bipartite and is distance Laplacian integral. Similarly, $\Gamma\left(\mathbb{Z}_{p_{1}^{n_{1}} p_{2}} n_{2}\right), n_{1}, n_{2} \geq 2$, contains the triangle $p_{1}^{n_{1}-1} p_{2}^{n_{2}} \sim p_{1} p_{2}^{n_{2}-1} \sim p_{1}^{n_{1}} p_{2}^{n_{2}-1} \sim p_{1}^{n_{1}-1} p_{1}^{n_{2}}$, so its zero divisor graph is not complete bipartite. Again, for $n=p^{2} q$ or $n=p q^{2}$, by Lemma 6, the zero divisor graph is not integral. Therefore, $\Gamma\left(\mathbb{Z}_{n}\right)$ is distance Laplacian integral only for $n=p^{2}, p q, 4 q$ with $p<q$ being primes.

Another consequence of Theorems 1 and 2 is the following.

Corollary 3. If $n$ is either prime or product of two distinct primes, then the smallest and largest distance Laplacian eigenvalue of $\Gamma\left(\mathbb{Z}_{n}\right)$ is the eigenvalue of quotient matrix. 


\section{Acknowledgements}

The authors are thankful to the anonymous referee for his valuable comments. The research of Pirzada S. is supported by SERB-DST, New Delhi under the research project number MTR/2017/000084.

\section{References}

[1] Anderson D.F., Livingston P.S. The zero divisor graph of a commutative ring. J. Algebra 1999, 217 (2), $434-447$. doi:10.1006/jabr.1998.7840

[2] Aouchiche M., Hansen P. Two Laplacians for the distance matrix of a graph. Linear Algebra Appl. 2013, 439 (1), 21-33. doi:10.1016/j.laa.2013.02.030

[3] Aouchiche M., Hansen P. Some properties of the distance Laplacian eigenvalues a graph. Czechoslovak Math. J. 2014, 64 (3), 751-761. doi:10.1007/s10587-014-0129-2

[4] Atiyah M.F., MacDonald I.G. Introduction to Commutative Algebra. Addison Wesley Publ., Boston, 1994.

[5] Chattopadhyay S., Patra K.L., Sahoo B.K. Laplacian eigenvalues of the zero divisor graph of the ring $\mathbb{Z}_{n}$. Linear Algebra Appl. 2020, 584, 267-286. doi:10.1016/j.laa.2019.08.015

[6] Cvetković D.M., Rowlinson P., Simić S. An Introduction to Theory of Graph spectra. Cambridge University Press, Cambridge, 2010. doi:10.1017/CBO9780511801518

[7] Ganie H.A. On distance Laplacian spectrum (energy) of graphs. Discrete Math. Algorithms Appl. 2020,12 (5), 2050061. doi:10.1142/S1793830920500615

[8] Horn R., Johnson C. Matrix Analysis. Cambridge University Press, Cambridge, 2013.

[9] Young M. Adjacency matrices of zero divisor graphs of integer modulo n. 2015, Involve 8 (5), 753-761. doi: 10.2140/involve.2015.8.753

[10] Pirzada S. An Introduction to Graph Theory. Universities Press, Orient BlackSwan, Hyderabad, 2012.

[11] Pirzada S., Rather B.A., Aijaz M., Chishti T.A. On distance signless Laplacian spectrum of graphs and spectrum of zero divisor graphs of $\mathbb{Z}_{n}$. Linear Multilinear Algebra 2020. doi:10.1080/03081087.2020.1838425

[12] Pirzada S., Rather B.A., Shaban R.U., Merajuddin. On signless Laplacian spectrum of the zero divisor graphs of the ring $\mathbb{Z}_{n}$. Korean J. Math. 2021, 29 (1), 13-24.

[13] Rather B.A., Pirzada S., Naikoo T.A., Shang Y. On Laplacian eigenvalues of the zero-divisor graph associated to the ring of integers modulo $n$. Mathematics 2021, 9 (5), 482. doi:10.3390/math9050482

[14] Stevanović D. Large sets of long distance equienergetic graphs. Ars Math. Contemp. 2009,2 (1), $35-40$. doi:10.26493/1855-3974.93.e75

Received 03.03.2020

Пірзада С., Ратхер Б.А., Хішті Т.А. Про дистаниійний лапласіановий спектр графів дільників нуля кільця $\mathbb{Z}_{n}$ // Карпатські матем. публ. - 2021. — Т.13, №1. - С. 48-57.

$\Delta$ ля скінченного комутативного кільця $\mathbb{Z}_{n}$ з одиничним елементом $1 \neq 0$ граф дільників нуля $\Gamma\left(\mathbb{Z}_{n}\right) \in$ простим зв'язним графом, множина вершин якого $є$ множиною ненульових дільників нуля, причому дві вершини $x$ та $y \in$ сусідніми тоді і тільки тоді, коли $x y=0$. Ми знаходимо дистанційний лапласіановий спектр графів дільників нуля $\Gamma\left(\mathbb{Z}_{n}\right)$ для різних значень $n$. Також ми отримуємо дистанційний лапласіановий спектр графа $\Gamma\left(\mathbb{Z}_{n}\right)$ для $n=p^{z}, z \geq 2$, у термінах лапласіанового спектра. Як наслідок ми визначаємо ті значення $n$, для яких граф дільників нуля $Г\left(\mathbb{Z}_{n}\right) \in$ дистанційним лапласіановим інтегралом.

Ключові слова і фрази: матриця Кірхгофа, матриця Кірхгофа відстаней, комутативне кільце, граф дільників нуля. 